\title{
Phenol levels during intralesional curettage and local adjuvant treatment of benign and low- grade malignant bone tumours
}

\author{
Suzan HM Verdegaal ${ }^{1 *}$, Jan den Hartigh², Pancras CW Hogendoorn ${ }^{3}$, Hugo FG Brouwers ${ }^{1}$ and \\ Antonie HM Taminiau'
}

\begin{abstract}
Background: Phenol is widely used for years as local adjuvant treatment for bone tumours. Despite its use for a long time, no information is available about the local concentration of phenol that is achieved in an individual patient, and the most sufficient and safe procedure to wash out the phenol after using it as local adjuvant.

Questions/purposes: 1. What is the initial local concentration of phenol in the tissue of the cavity wall after the application of phenol? 2. How quickly is phenol $85 \%$ diluted by washing the bone cavity with ethanol $96 \%$ solution? 3. Is the degree and speed of dilution influenced by the size of the cavity? 4. How many times should the cavity be rinsed to obtain sufficient elimination of phenol?

Methods: A basic science study was performed at respectively 16 and 10 patients, treated by intralesional curettage and adjuvant therapy for low-grade central chondrosarcoma of bone. Test 1:in 16 patients ten samples were collected of the mixture of phenol and ethanol from the bone cavity. Test 2:in ten patients, two biopsy samples were taken from the cavity wall in the bone during surgery.

Results: Phenol concentrations had wide variety in different patients, but all decreased by rinsing with ethanol.

Conclusions: Ethanol $96 \%$ is effective to wash out local applicated phenol, by rinsing the bone cavity six times. The local concentration of phenol diminishes to an acceptable concentration of $0.2 \%$. This study provides new insights to safely further improve the surgical technique of intralesional treatment of bone tumours.
\end{abstract}

\section{Background}

In the surgical treatment of benign bone tumours, intralesional curettage has been performed throughout the past century. Over the recent years, its use has been extended to low-grade intramedullary malignant tumours in some instances. Unfortunately, this surgical technique has the risk of local recurrence from tumour cells that may be left behind. For this reason curettage was supplemented by the use of a local adjuvant, such as phenol, liquid nitrogen or bone cement (poly methyl methacrylate, PMMA). Various studies have demonstrated that by using adjuvant, the results of local therapy have been greatly improved [1-4].

\footnotetext{
* Correspondence: shmverdegaal@hotmail.com

'Department of Orthopaedic Surgery, Leiden University Medical Center, Leiden, The Netherlands

Full list of author information is available at the end of the article
}

With regard to liquefied phenol, only a few documented studies have been published, despite of its routine use over a long period in orthopaedic practice. Previously, we were able to show that in case of low-grade chondrosarcoma, phenol is able to kill tumour cells in-vitro already at a concentration of 3\% [5]. In clinical settings, liquefied phenol is known to reduce local recurrence rates in different benign bone tumours such as aneurysmal bone cysts, chondroblastoma and giant cell tumours from $\mathbf{4 1 \%}$ to $\mathbf{7 - 9 \%}[1,2]$. Liquefied phenol, containing 82.0 to $86.5 \% \mathrm{w} / \mathrm{w}$ phenol in water, is a colourless or faintly coloured liquid. It may be used as a preservative in pharmaceuticals and chemicals, but is also widely used in household products. Liquefied phenol is readily absorbed via inhalation, ingestion and dermal contact, causing both local and systemic toxicity. The elimination half-life ranges from 1-14 hours [6]. It is
C Biomed Central

() 2012 Verdegaal et al; licensee BioMed Central Ltd. This is an Open Access article distributed under the terms of the Creative Commons Attribution License (http://creativecommons.org/licenses/by/2.0), which permits unrestricted use, distribution, and reproduction in any medium, provided the original work is properly cited. 
eliminated in the urine, mainly as sulfate and glucuronide conjugates. Liquefied phenol causes cell-wall disruption, precipitation, denaturation of proteins and coagulation necrosis. Aqueous solutions as dilute as $10 \%$ may be corrosive.

Clinical symptoms of phenol after ingestion may include local corrosion with pain, nausea, vomiting and diarrhea [6]. Systemic toxicity may consist of CNS depression, circulatory and respiratory failure, pulmonary edema and hepatic and renal injury. Poisoning may occur from skin contact, especially from wounds. Applied to the skin, phenol causes blanching and corrosion in a concentration of $1-2 \%$, depending on the exposure time.

In case of surgery, a bone-window is created in order to perform a subsequent curettage of the tumour. To ensure that the cavity that has been formed is microscopically tumour-free, the walls are cauterised with an $85 \%$ solution of liquefied phenol. Given that liquefied phenol has toxic and potentially carcinogenic properties when used systemically over a longer period, the cavity is then rinsed a number of times with a $96 \%$ solution of ethanol. The cavity is filled with allograft bone-chips and the bone-window, which has also been rinsed with liquefied phenol and ethanol, is placed back in position. The above mentioned surgical technique has our preference given its advantage to induce minimal local damage and a biological reconstruction. Alternative products to fill the resultant defect after curettage are PMMA, auto graft bone chips or synthetic bone materials.

Regarding the surgical technique, some questions remain:

1. What is the initial local concentration of phenol in the tissue of the cavity wall after the application of liquefied phenol $85 \%$ ?

2. How quickly is the liquefied phenol $85 \%$ diluted by washing the bone cavity with ethanol $96 \%$ solution?

3 . Is the degree and speed of dilution influenced by the size of the cavity?

4. How many times should the cavity be rinsed to obtain sufficient elimination of phenol?

Answering these questions will further elucidate the safe practice of phenol-assisted, intralesional curettage of low grade malignant intramedullary bone tumours.

\section{Patients and methods Patients}

16 patients (5 male, 11 female) with a median age of 48 (range 26-70) at time of surgery, were treated for grade I chondrosarcoma. The tumours are predominantly located in the proximal humerus $(7 / 16,44 \%)$ and the distal femur $(5 / 16,31 \%)$ (Table 1). The lesions were histologically classified by an experienced pathologist according to the recently published consensus criteria [7] and graded according to the Evans' grading [8].

\section{Clinical setting}

Following a trial on three patients to test the feasibility of the study, 16 patients, suspected with grade I chondrosarcoma following X-ray and dynamic Gd-MRI [9], were treated according to the surgical technique described above. Two tests were performed. Test 1 was done on all 16 patients, test 2 on 10 patients. Before performing surgery including these tests, all patients were informed and gave their approval. Patient material was used in a coded fashion according to the local ethical regulation and in accordance to the national ethical guidelines (Dutch organisation of scientific societies FEDERA; "Code for proper secondary use of human tissue" in the Netherlands).

\section{Treatment protocol}

\section{Test 1}

Depending on the size of the cavity, 2 to $4 \mathrm{ml}$ of liquefied phenol $85 \%$ was applied with one or more small gauzes at the inner surface of the wall for 3 minutes after curettage of the bone tumour. Then the cavity was filled with an ethanol $96 \%$ solution. The volume of ethanol differed depending on the size of the cavity. Subsequently, this mixture of phenol and ethanol was extracted by syringe and sealed immediately in a polypropylene container. This procedure was repeated ten times and labelled accordingly (sample numbers I to X).

\section{Test 2}

During surgery two biopsies were taken. The first biopsy (A)was taken after the cavity had been swabbed with the $85 \%$ phenol solution. A second biopsy (B) was taken after the cavity had been rinsed thoroughly ten times with ethanol, as described above (Table 2).

These biopsies (A and B), plus the ten flush solutions (I to $\mathrm{X}$ ) were then further investigated to determine the concentration of phenol.

\section{The surface area of the cavity}

It is necessary to approximate the shape of the cavity, because the actual surface area does not fit in any standard mathematical model and therefore cannot be measured with absolute precision. Therefore we projected a cylinder over the lesion using the largest diameter of the cartilaginous tumour of the long bones, measured in three dimensions on the pre-operative MRI. A standard formula was used to calculate the inner surface of this cylindrical curetted bone cavity: $2 \pi r(r+h)$. $R$ represents the average of half the width (mediolateral) and half the depth (anteroposterior), which often corresponds with the inside diameter of the bone. $H$ is the length of the tumour taken in a craniocaudal direction.

\section{Sampling}

The flush solutions, from test 1 , and the bone biopsies, from test 2 , were stored in a refrigerator until analysis. 
Table 1 Patient information, tumour volume and cavity surface

\begin{tabular}{|c|c|c|c|c|c|}
\hline Patient & Age/Gender & PA & Location & Tumour volume $(\mathrm{cm} 3)$ & Cavity surface $(\mathrm{cm} 2)$ \\
\hline 1 & $41 / \mathrm{M}$ & CHS I & Proximal humerus & 27 & 73,4 \\
\hline 2 & 39/M & CHS I & Distal femur & 48,1 & 74,2 \\
\hline 3 & $40 / F$ & CHS I & Distal tibia & 9,2 & 24,6 \\
\hline 4 & $411 \mathrm{M}$ & CHS I & Proximal humerus & 26,3 & 62,3 \\
\hline 5 & $45 / F$ & CHS I & Proximal humerus & 40,8 & 72,6 \\
\hline 6 & $70 \mathrm{ff}$ & CHS I & Distal femur & 10,9 & 62,3 \\
\hline 7 & $55 / M$ & CHS I & Distal femur & 39,4 & 74,6 \\
\hline 8 & $59 / F$ & CHS I & Proximalhumerus & 14,7 & 33,4 \\
\hline 9 & $49 / F$ & CHS ॥ & Proximal humerus & 9,7 & 25,8 \\
\hline 10 & $48 / F$ & CHS I & Distal femur & 33 & 58,8 \\
\hline 11 & $67 / F$ & CHS I & Distal tibia & 5,2 & 16,8 \\
\hline 12 & $44 / F$ & CHS I & Proximal humerus & 25,8 & 49,4 \\
\hline 13 & $33 / \mathrm{Nl}$ & CHS I & Metacarpal & $\vee 4,4$ & 15,3 \\
\hline 14 & $50 / F$ & CHS I & Humerus & 44 & 74,5 \\
\hline 15 & $55 / F$ & CHS I & Proximal humerus & 32 & 59,3 \\
\hline 16 & $26 / F$ & CHS I & Distal femur & 20 & 51,5 \\
\hline
\end{tabular}

Characteristics of the 16 patients where the tests during surgery were performed. Depending on the location of the chondrosarcoma, the cavity surface varies.

Flush solutions were analyzed as such, or after dilution (see phenol analysis). Bone biopsies were dispersed in $5.0 \mathrm{ml}$ ethanol $96 \% \mathrm{v} / \mathrm{v}$; an aliquot of this solution was analyzed.

\section{Phenol analysis}

The phenol concentration of the ethanolic flush solutions was determined by High Performance Liquid Chromatography (HPLC) with spectrophotometric detection. Briefly, chromatographic analysis of the samples (diluted with ethanol to fit within the concentration range of the calibration curve, if necessary) was performed on a silica reversed phase column (Nucleosil C18, $100 \times 3 \mathrm{~mm}, 5 \mu \mathrm{m}$ particle size) with a mobile phase consisting of $25 \mathrm{mM}$ phosphate buffer containing $0,5 \%$ triethylamine $\mathrm{pH} 3.0$ and acetonitrile $(83+17, \mathrm{v} /$ v). The column flow rate was $0.4 \mathrm{ml} / \mathrm{min}$ and detection was performed at $212 \mathrm{~nm}$. The injection volume was 10 $\mu \mathrm{l}$. The phenol concentrations in the flush solutions were calculated from calibration graphs obtained by simultaneous analysis of phenol standard solutions in ethanol $96 \% \mathrm{v} / \mathrm{v}$ in the concentration range $30-1500$ ppm phenol. Up to $500 \mathrm{ppm}$ there was a linear relation between response and concentration; above 500 ppm a quadratic function had to be used. Inter-day reproducibility showed a coefficient of variation of $5.1 \%$ at a concentration level of $50 \mathrm{ppm}$ and $10.6 \%$ at $5 \mathrm{ppm}(\mathrm{n}=6)$. The lower limit of quantitation (LLOQ) was $5 \mathrm{ppm}$. Basic descriptive statistics were employed.

\section{Results}

Test 1

The phenol concentrations measured in the flush solutions ranged from 29 to 20974 ppm. Large inter-patient differences were observed. Figure 1 shows a graphical presentation of the decay in phenol concentration over

Table 2 Concentrations of phenol in sixteen patients during washing out phenol with ethanol

\begin{tabular}{cccccccccccccccccccc}
\hline & $\mathbf{1}$ & $\mathbf{2}$ & $\mathbf{3}$ & $\mathbf{4}$ & $\mathbf{5}$ & $\mathbf{6}$ & $\mathbf{7}$ & $\mathbf{8}$ & $\mathbf{9}$ & $\mathbf{1 0}$ & $\mathbf{1 1}$ & $\mathbf{1 2}$ & $\mathbf{1 3}$ & $\mathbf{1 4}$ & $\mathbf{1 5}$ & $\mathbf{1 6}$ \\
\hline I & 929 & 2435 & 8032 & 3264 & 9661 & 13000 & 289 & 1300 & 1500 & 1500 & 1500 & 2299 & 20974 & 1239 & 373 & 160 \\
II & 449 & 900 & 2889 & 730 & 1780 & 563 & 193 & 787 & 734 & 1500 & 1478 & 816 & 13932 & 889 & 247 & 118 \\
III & 177 & 469 & 2169 & 293 & 1097 & 201 & 555 & 473 & 329 & 539 & 1199 & 523 & 4652 & 644 & 159 & 97 \\
IV & 92 & 313 & 1019 & 83 & 944 & 119 & 156 & 418 & 249 & 195 & 495 & 292 & 1739 & 465 & 82 & 66 & 195 \\
V & 84 & 185 & 797 & 106 & 632 & 162 & 167 & 313 & 150 & 160 & 185 & 261 & 1932 & 407 & 77 & 89 \\
VI & 69 & 189 & 522 & 73 & 312 & 126 & 146 & 289 & 127 & 87 & 249 & 223 & 1287 & 307 & 82 & 54 \\
VII & 65 & 128 & 400 & 49 & 168 & 101 & 98 & 191 & 97 & 126 & 157 & 160 & 805 \\
VIII & 65 & 146 & 381 & 72 & 227 & 72 & 114 & 126 & 97 & 116 & 217 & 140 & 900 & 215 & 65 & 39 \\
IX & 42 & 325 & 294 & 59 & 392 & 138 & 52 & 124 & 110 & 57 & 178 & 111 & 597 & 162 & 87 & 42 \\
$X$ & 105 & 217 & 279 & 47 & 200 & 54 & 64 & 98 & 88 & 60 & 143 & 117 & 417 & 182 & 60 & 29 \\
\hline
\end{tabular}

Results of 16 patients (1-16); after using phenol $85 \%$ as adjuvant therapy, ethanol $96 \%$ was used to wash out the phenol; sample I to $\mathrm{X}$ show ppm of phenol in ethanol; Note that the bold results are not reliable or higher measured level than in reality. 


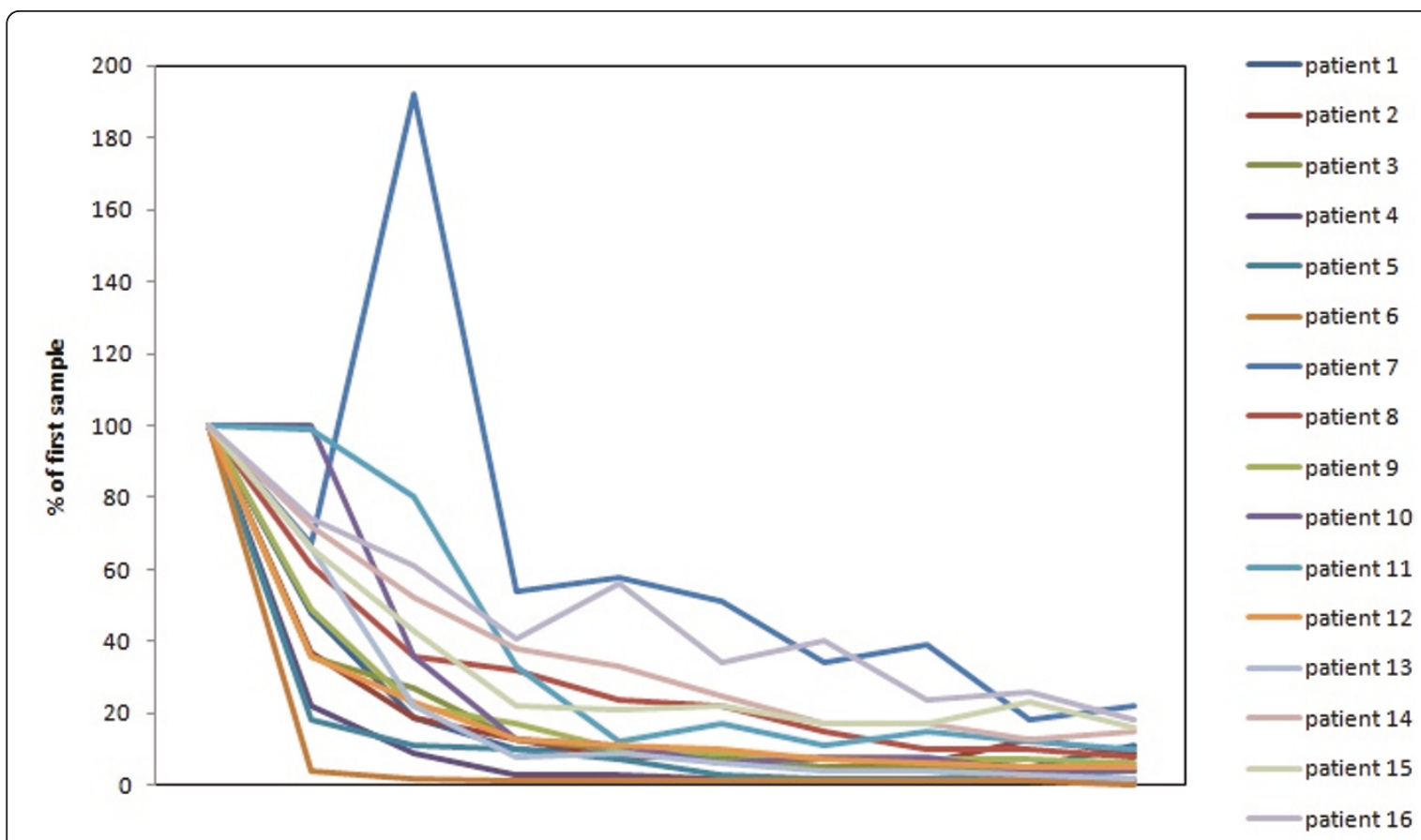

samples I to $\mathrm{X}$

Figure 1 Concentrations of phenol in ethanol. Results of ten samples of ethanol with phenol in 16 patients; samples II to X are reproduced as the fraction of the phenol concentration, measured in the first sample.

time observed for each patient, expressed as a percentage of the concentration measured in the first flush sample.

After washing the bone cavity five times with ethanol $96 \%$, in most of the patients the phenol levels measured are $<280 \mathrm{ppm}$.

Patient number 7 has one very high concentration of phenol in sample III (Table 2). We can only explain this as a sample error, because from sample IV to $\mathrm{X}$ the concentration decreases.

\section{Test 2}

In Table 3 the phenol concentrations found in the biopsies, dispersed in $5 \mathrm{ml}$. ethanol, are presented. For all patients the phenol concentration before (A) and after flushing the cavity with ethanol 96\% (B) is shown. Remarkable differences were measured, quantative as well as in reducing the concentration of phenol washed out by ethanol.

\section{Tumour volume}

Median volume of the cavity is $24.4 \mathrm{~cm} 3$ (range 4-48), and depends on the location of the tumour. The smallest volumes are measured in the distal tibia and fifth metacarpal bone.

No correlation is seen between the concentrations of phenol in ethanol in large or small cavities (Figure 2).

\section{Discussion}

The use of chemical cauterization for benign and lowgrade malign bone tumours was first proposed by Bloodgood [10]. Although phenol is used for many years as adjuvant therapy in the intralesional treatment of benign and low-grade malignant bone tumours, no information is available about the local concentration of phenol in

Table 3 Concentrations of phenol solved in $5 \mathrm{ml}$. of ethanol during surgery

\begin{tabular}{lrrrrrrrrr}
\hline & $\mathbf{8}$ & $\mathbf{9}$ & $\mathbf{1 0}$ & $\mathbf{1 1}$ & $\mathbf{1 2}$ & $\mathbf{1 3}$ & $\mathbf{1 4}$ & $\mathbf{1 5}$ & $\mathbf{1 6}$ \\
\hline biopsy A (ppm) & 1598 & 888 & 168 & 479 & 1043 & 25 & 572 & 94 & 144 \\
biopsy B (ppm) & 408 & 418 & 39 & 106 & 636 & 17 & 587 & 11 & 18 \\
\hline
\end{tabular}

Results of phenol samples 9 patients (8-16); first bone biopsy (A) was taken after application phenol $85 \%$. After washing ten times with ethanol $85 \%$ the second biopsy (B) was performed. The concentrations of phenol (ppm) in this table are solved in $5 \mathrm{ml}$. of ethanol. 


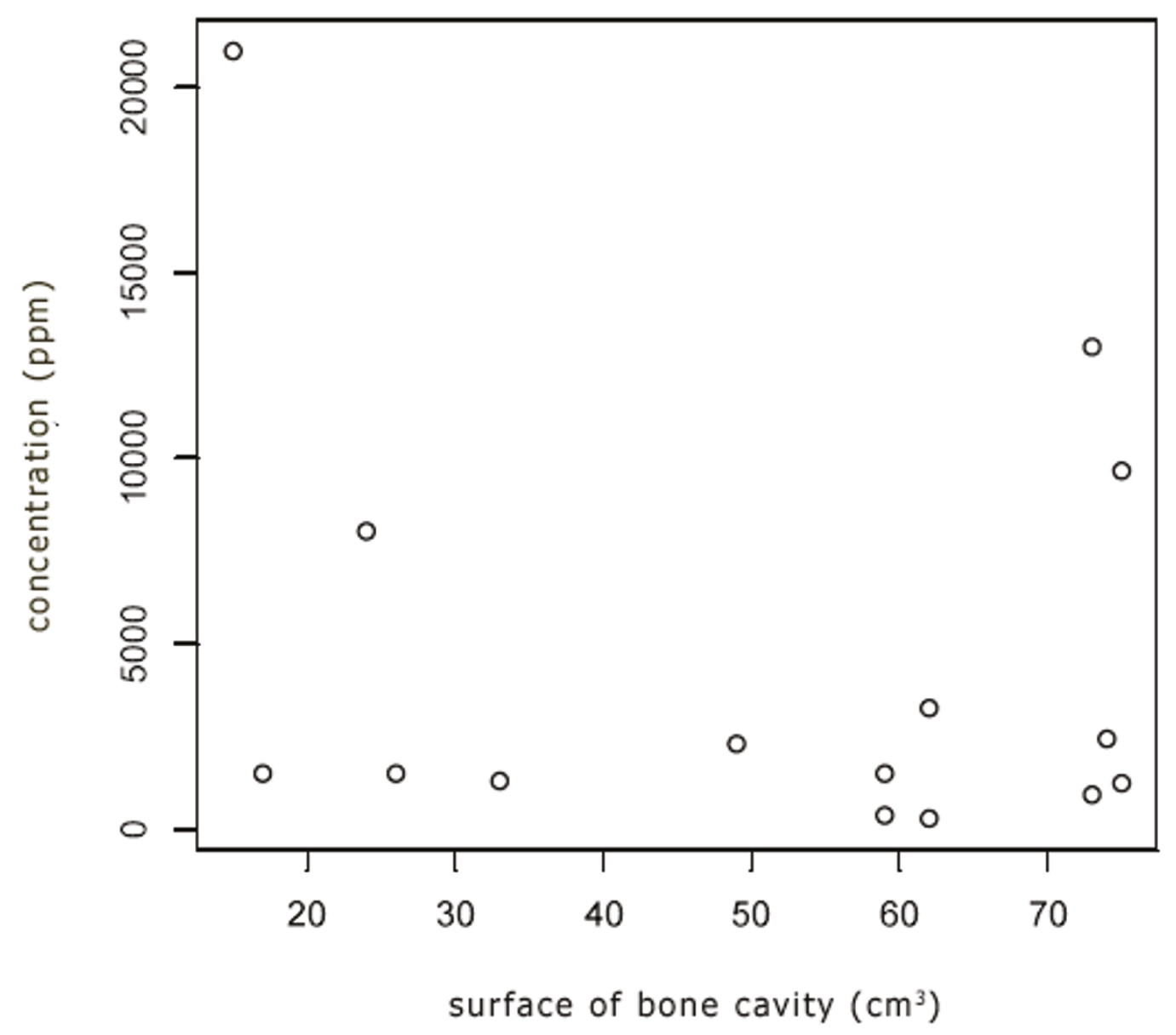

Figure 2 Box Plot of concentration phenol and surface of bone cavity. Relation between concentrations of phenol measured in ethanol $(\mathrm{ppm})$ and surface of the bone cavity. No correlation is seen between the concentrations of phenol in ethanol in large or small cavities.

individuals, the most sufficient and safest procedure to wash out the phenol after the local use.

Some surgeons have a dislike to the use of phenol as adjuvant, due to the possible predisposition to infection, the inhibition of the incorporation of grafts, systemic toxic effects on patients, and possible adverse effects on personnel in the operating theatre.

In our opinion, the highest estimated risk for using phenol is the people who work with it in the operating room. To prevent skin contact, using the routine measures such as by wearing proper protection to hands and face is sufficient. In case of skin contact, the skin should be irrigated with polyethylene glycol (PEG) 400 solution, isopropanol $70 \%$ or, if not available, with water [10-14]. During surgery, there is a risk of fire, with the evaporation of ethanol and simultaneous use of electric cauterization devices, as this can cause a spark. Therefore, we strongly recommend disconnecting the electric devices from the moment phenol and ethanol bottles are to be opened.
The two biopsies A and B were taken from the cavity wall before starting washing out and after rinsing 10 times with ethanol 96\%. In biopsy B, the quantity of phenol is diminished, as expected, but still there is quite a concentration left. This is due to the fact, that phenol is very lipophylic and therefore not easy to remove with ethanol 96\%. The results also show remarkable differences in a quantative way. In these patients, tumour volume, differences in dilution by wound fluid and blood in the cavity, and differences in the $\mathrm{ml}$ of phenol applicated explain these results. In patient 14, the concentration even increases a little bit after washing out with ethanol. In this patient, it seems that it is hard to wash out the local phenol from the cavity wall.

Concerning the initial local concentration of phenol in the cavity wall after application of phenol, two biopsies were curetted. Test 2 shows the concentrations in biopsy A and B. Starting with $85 \%$ of liquefied phenol, the mean initial local concentration of phenol measured was 557 
ppm (range 25-1598, biopsy A, Table 3). The measurements have a large variation.

Patient 13 suffered from a small chondrosarcoma of the metacarpal bone. The volume of the tumour was $4,4 \mathrm{~cm}^{3}$. Biopsy A and B show 25 and 17 ppm phenol respectively. Nevertheless, the first sample in test 1 has a very high concentration of phenol in ethanol, 20974 ppm. The possible explanation of these controversial measurements is, that the location where the biopsy A and B were taken from the metacarpal bone can almost only be cortical border of the tumour. As cortical bone won't absorb phenol very well, the concentrations in biopsy A and B are low.

To investigate the effect of ethanol to dilute the applicated phenol, 10 samples in 16 patients were collected. In test 1 , in all 16 cases the highest concentration of phenol was measured in the first sample. However, the absolute values differ. Despite the fact that the same procedure was performed in all cases, no procedure is the same given the different volumes of the tumours varying from 4 to $48 \mathrm{~cm}^{3}$. In patient number seven, the III ${ }^{\text {rd }}$ sample shows an increase of the concentration phenol. From the $\mathrm{IV}^{\text {th }}$ sample, the line decrease further on sample II. The III $^{\text {rd }}$ sample must be considered to be a sample error. In these tests, the cavity was filled with ethanol ten times, and phenol concentrations were measured. After washing the cavity 6 times, in 10 of 16 patients the fraction of the initial concentration of phenol is $<10 \%$. The quantative concentration of phenol is $<1300 \mathrm{ppm}$ in all cases, which means an estimated concentration of phenol of $<0,2 \%$.

The degree of dilution is not influenced by the size of the cavity after curettage. However, it is remarkable that the highest concentrations in the first samples of test 1 all concerned small tumour volumes. This can be explained by the relatively small volume of ethanol where the phenol is diluted in.

We did not specifically study systemic effects of phenol exposure in our patients; however, on retrospective review, no adverse effects or secondary malignancies were noted.

In the few studies that described measurements of phenol, this was done by taking samples of urine, as liquefied phenol is eliminated by the kidneys. However, it is hard to define a 'thresh hold' for the toxic level.

This study shows that the adverse effects on the whole body due to the use of liquefied phenol as adjuvant in the intralesional curettage of benign and low-grade malignant bone tumours are reduced to safe concentrations by washing phenol out by ethanol. Phenol is a safe adjuvant when used in a proper way, taking the above mentioned statements into account. Washing the cavity six times with ethanol $96 \%$ will be sufficient to diminish the local concentration of liquefied phenol to an acceptable concentration of $<0.2 \%$.

\section{Acknowledgements}

We would like to thank A. Verhaegen for performing the laboratory tests, E. van Zwet for his help with statistical analysis; and the EuroBoNeT consortium, an European Commission granted Network of Excellence for studying the pathology and genetics of bone tumours for their support.

\section{Author details}

${ }^{1}$ Department of Orthopaedic Surgery, Leiden University Medical Center, Leiden, The Netherlands. ${ }^{2}$ Department of Clinical Pharmacy and Toxicology, Leiden University Medical Center, Leiden, The Netherlands. ${ }^{3}$ Department of Pathology, Leiden University Medical Center, Leiden, The Netherlands.

\section{Authors' contributions}

SHMV participated in the conception and design of the study, performed samples during surgery, performed analysis and interpretation of the data, and drafted the paper. JdH participated in the conception and design of the study, the technical design of the phenol analysis including performing of the laboratory tests, and interpretation of the data. HFGB contributed in acquisition and interpretation of data, and performing the tables and figures. PCWH participated in the conception and design of the study, the analysis and interpretation of the data. AHMT participated in the conception and design of the study, performed the surgery and took samples during surgery and performed analysis and interpretation of the data. All authors read and approved the final manuscript.

\section{Conflicts of interests}

The authors declare that they have no competing interests.

Received: 20 January 2012 Accepted: 26 March 2012

Published: 26 March 2012

\section{References}

1. Capanna R, Sudanese A, Baldini N, Campanacci M: Phenol as an adjuvant in the control of local recurrence of benign neoplasms of bone treated by curettage. Ital J Orthop Traumatol 1985, 11:381-388.

2. Durr HR, Maier M, Jansson V, Baur A, Refior HJ: Phenol as an adjuvant for local control in the treatment of giant cell tumour of the bone. Eur J Surg Oncol 1999, 25:610-618.

3. Szendroi M: Adjuvant therapy (phenol, bone cement) in giant cell tumors. Z Orthop Ihre Grenzgeb 1992, 130:95-98.

4. Verdegaal SHM, Brouwers HFG, Zwet van EW, Hogendoorn PCW, Taminiau AHM: Low-grade chondrosarcoma of the long bones treated by intralesional curettage followed by the application of phenol, ethanol and bone grafting. J Bone Joint Surg Am, accepted for publication December 23rd 2011.

5. Verdegaal SH, Corver WE, Hogendoorn PC, Taminiau AH: The cytotoxic effect of phenol and ethanol on the chondrosarcoma-derived cell line OUMS-27: an in vitro experiment. J Bone Joint Surg Br 2008, 90:1528-1532.

6. Bentur Y, Shoshani O, Tabak A, Bin-Nun A, Ramon Y, Ulman Y, et al: Prolonged elimination half-life of phenol after dermal exposure. J Toxicol Clin Toxicol 1998, 36:707-711.

7. Eefting D, Schrage YM, Geirnaerdt MJ, Le CS, Taminiau AH, Bovee JV, et al: Assessment of interobserver variability and histologic parameters to improve reliability in classification and grading of central cartilaginous tumors. Am J Surg Pathol 2009, 33:50-57.

8. Evans HL, Ayala AG, Romsdahl MM: Prognostic factors in chondrosarcoma of bone: a clinicopathologic analysis with emphasis on histologic grading. Cancer 1977, 40:818-831.

9. Geirnaerdt MJ, Hogendoorn PC, Bloem JL, Taminiau AH, Van der Woude HJ: Cartilaginous tumors: fast contrast-enhanced MR imaging. Radiology 2000, 214:539-546.

10. Bloodgood JC: Benign bone cysts, ostitis fibrosa, giant-cell sarcoma and bone aneurism of the long pipe bones. Ann Surg 1910, 52:145-185.

11. Caravati EM: Household Products. In Medical Toxicology. [201]. Edited by: Dart RC. Lippincott: Williams and Wilkins; 2004:1294-1303, Ref Type: Edited Book.

12. Horch R, Spilker G, Stark GB: Phenol burns and intoxications. Burns 1994, 20:45-50.

13. Hunter DM, Timerding BL, Leonard RB, McCalmont TH, Schwartz E: Effects of isopropyl alcohol, ethanol, and polyethylene glycol/industrial 
methylated spirits in the treatment of acute phenol burns. Ann Emerg Med 1992, 21:1303-1307.

14. Monteiro-Riviere NA, Inman AO, Jackson H, Dunn B, Dimond S: Efficacy of topical phenol decontamination strategies on severity of acute phenol chemical burns and dermal absorption: in vitro and in vivo studies in pig skin. Toxicol Ind Health 2001, 17:95-104.

doi:10.1186/2045-3329-2-10

Cite this article as: Verdegaal et al:: Phenol levels during intralesional curettage and local adjuvant treatment of benign and low-grade malignant bone tumours. Clinical Sarcoma Research 2012 2:10.

Submit your next manuscript to BioMed Central and take full advantage of:

- Convenient online submission

- Thorough peer review

- No space constraints or color figure charges

- Immediate publication on acceptance

- Inclusion in PubMed, CAS, Scopus and Google Scholar

- Research which is freely available for redistribution

Submit your manuscript at www.biomedcentral.com/submit
Ciomed Central 